\title{
Coherence Length Calculation for the Outcoupled Beam of Atom Laser
}

\author{
Abdul-Basit A. Saleh ${ }^{\dagger}$ and Noori H. N. Al-Hashmiy \\ ${ }^{\dagger}$ Department of Physics, Faculty of Science, Sana'a University, PO Box 8211, Sana'a, Yemen \\ ${ }^{\ddagger}$ Department of Physics, College of Education, University of Basrah, Basrah, Iraq
}

Received on 12 April, 2007

\begin{abstract}
We use the theory of coupled Gross-Pitaevskii equations to derive an expression for the current density of the propagating atoms coupled out of the condensate under the influence of gravity. By describing the propagation vector of the atoms and using a theoretical definition of the coherence length, we calculate the coherence lengths of ${ }^{37} \mathrm{~K},{ }^{141} \mathrm{Ce}$, and ${ }^{249} \mathrm{Fm}$ atom lasers numerically. Our calculation method is verified with the available experimental and theoretical data in the literature. The results showed a very good agreement.
\end{abstract}

Keywords: Atom lasers; Boson systems; Optical cooling of atoms; Trapping

\section{INTRODUCTION}

The observation of Bose-Einstein condensate (BEC) in dilute alkali gases in 1995 [1-3], confirmed by the award of the 2001 Nobel Prize to the pioneers [4, 5], was the key to the recent advancements in atomic, optical, and molecular physics. That observation has lead to a new field of research that deals with a propagating and coherent beam of matter waves, known as an atom laser. These matter waves were proven to interact with each other as they propagate, and their focused beams have shown interference patterns associated with phase coherence $[6,7]$.

In this paper, we use the theory of coupled Gross-Pitaevskii (GP) equations to calculate a general expression for the current density of the propagating beam of atoms coupled out of the condensate due to the mean-field potential under the influence of gravity. We calculate the coherence lengths of ${ }^{37} \mathrm{~K}$, ${ }^{141} \mathrm{Ce}$, and ${ }^{249} \mathrm{Fm}$ atom lasers by solving the definition of the coherence length as proposed by Castellanos and Lopez [8]. The calculations are made numerically using a computer programme created in FORTRAN. To verify the validity of our calculation method, first we applied it to three other atoms $\left({ }^{23} \mathrm{Na},{ }^{87} \mathrm{Rb}\right.$, and $\left.{ }^{7} \mathrm{Li}\right)$ of known coherence lengths in the literature $[8,9]$. The results showed a very good agreement.

In section 2, we use the coupled GP equations to review and derive the outcoupled wave function $\psi_{0}$ for the untrapped atoms, taking into account the influence of gravity and the weak coupling limit relevant to the quasi-continuous atom laser [10]. In section 3, we calculate the current of the propagating atoms coupled out of the condensate. Considering a pair of atoms, we derive the propagation vector of the travelling atoms and obtain a definition for the coherence length of the atom laser in section 4 . Solving this equation of the coherence length numerically, we describe our calculation method of the coherence length for the ${ }^{37} \mathrm{~K},{ }^{141} \mathrm{Ce}$, and ${ }^{249} \mathrm{Fm}$ atom lasers in section 5, comparing and discussing our results with the available data in the literature and making a conclusion on the validity of our calculation method.

\section{THE COUPLED GROSS-PITAEVSKII EQUATIONS THEORY}

Output coupling is essential in turning the trapped BEC into an atom laser. The output coupler consists of a monochromatic resonant radio frequency (rf) field of frequency $\omega_{\mathrm{rf}}$, which has been used to transfer the confined condensate of atoms from the trapped state to untrapped magnetic states, enabling the atoms to leave the trap under the influence of gravity with the generation of a coherent atom laser [11]. Gravity determines the propagation direction of the extracted atom laser as well as its amplitude and its phase. Theoretically, the output coupling from BEC is governed by Gross-Pitaevskii (GP) equations for the wave functions of the condensate in the trapped and untrapped magnetic states, coherently coupled by an external electromagnetic field. That is, the field associated with the BEC obeys equations of quantum nature which reduce, for the condensate, to the following time-dependent GP equation (see [12] and references therein).

$$
i \hbar \frac{\partial \psi_{m}(r, t)}{\partial t}=\left(-\frac{\hbar^{2}}{2 M} \nabla^{2}+V_{\text {trap }}+U_{0}\left|\psi_{m}(r, t)\right|^{2}\right) \psi_{m}(r, t)
$$

Mathematically, this equation is also the coupled timedependent non-linear Schrodinger equation for the condensate wave function $\psi_{m}(r, t)$. Here $U_{0}=\frac{4 \pi \hbar^{2} a}{M}$ is the interaction coupling constant, where $a$ is the scattering length, and $M$ is the atomic mass. Considering ${ }^{87} \mathrm{Rb}$ in the hyperfine level $F=1$ at $T=0 K$, with $m=-1,0,+1$ representing the three Zeeman sublevels, where $m=0$ and $m=+1$ states refer to the untrapped and the repelled atoms, respectively, and the $m=-1$ state refers to the trapped atoms in the magnetic potential [10]:

$$
V_{\text {trap }}=\frac{1}{2} M\left(\omega_{x}^{2} x^{2}+\omega_{\perp}^{2} y^{2}+\omega_{\perp}^{2} z^{2}\right)
$$

Here, $\omega_{x}$ is the frequency of the trap in the $x$ direction and $\omega_{\perp}$ is the frequency of the trap in the $y-z$ plane. The extraction of atoms from the trapped BEC under the influence of gravity is treated in the weak coupling regime [13]. In this regime, the coupling into the $m=+1$ state is neglected and the coupling strength is so small that the populations $N_{m}$ of the three sublevels satisfy the inequality $N_{+1} \ll N_{0} \ll N_{-1}$. The BEC wave functions $\psi_{m}(t)=\left\langle\psi_{m}(t)\right\rangle \exp \left(-i m \omega_{\mathrm{rf}} t\right)$, under the rotating wave approximation in the hyperfine level $F=1$, satisfy the following pair of coupled GP equations: 


$$
\begin{gathered}
i \hbar \frac{\partial \psi_{-1}(r, t)}{\partial t}=\left[\hbar \delta_{\mathrm{rf}}+\frac{p^{2}}{2 M}+V_{\text {trap }}+U_{0}\left|\psi_{-1}(r, t)\right|^{2}\right] \psi_{-1}(r, t)+\frac{\hbar \Omega}{2} \psi_{0}(r, t) \\
i \hbar \frac{\partial \psi_{0}(r, t)}{\partial t}=\left[\frac{p^{2}}{2 M}+M g z+U_{0}\left|\psi_{-1}(r, t)\right|^{2}\right] \psi_{0}(r, t)+\frac{\hbar \Omega}{2} \psi_{-1}(r, t)
\end{gathered}
$$

where the coupling constant $\hbar \Omega=\frac{\mu_{\mathrm{B}} B_{\mathrm{rf}}}{2 \sqrt{2}}$ refer to the Rabi frequency due to the magnetic field $B_{\mathrm{rf}}$ of the trap, $\hbar \delta_{\mathrm{rf}}=$ $V_{\text {off }}-\hbar \omega_{\text {rf }}$ is the detuning from the bottom of the trap, $\mu_{\mathrm{B}}$ is Bohr magneton, $g$ is the gravitational acceleration, and $p$ is the particles momentum. Here, the total condensate density $n(r, t)$ has been replaced by the modulus of the wave function $\left|\psi_{-1}(r, t)\right|^{2}$.

Gravity results in a sag of the trapped condensate. The displacement of the minimum of the external potentials away from the trap centre at $z=0$ depends on $m$ and is given by $z_{\text {sag }}=g / \omega_{\perp}^{2}$. The zero of energy is chosen at $z=0$ in the $m=0$ state so that the level splitting at the bottom of the trap is given by:

$$
V_{\mathrm{off}}=\frac{\mu_{\mathrm{B}} B_{\mathrm{off}}}{2}+\frac{M g^{2}}{2 \omega_{\perp}^{2}}
$$

where $B_{\text {off }}$ is the offset magnetic field of the trap. For a manybody interacting system, like trapped BEC, mean-field approaches using GP type equations are usually developed to overcome the problem of solving the many-body Schrodinger equation [12-15]. GP equations provide an understanding of the main features of the condensation and are known in their direct treatment of the interactions between particles. Accordingly, equations (3) and (4) are outcoupled within the formalism of the mean-field theory, obtaining for the outcoupled atom laser wave function the following expression [10]:

$$
\psi_{0}(r, t) \approx-\sqrt{\pi} \frac{\hbar \Omega}{M g l} \phi_{-1}\left(x, y, z_{\mathrm{res}}\right) \frac{\exp \left[i \frac{2}{3}\left|\xi_{\mathrm{res}}\right|^{\frac{3}{2}}\right]-i \frac{E_{-1} t}{\hbar}}{\sqrt{\left|\xi_{\mathrm{res}}\right|^{\frac{1}{2}}}} F\left(t, t_{\mathrm{fall}}\right)
$$

Here, $\phi_{-1}\left(x, y, z_{\text {res }}\right)$ is the time dependent ground state of the condensate, with energy equals to the chemical potential $\mu$ given by $\mu=\left(\frac{\hbar \omega_{\mathrm{rf}}}{2}\right)\left(\frac{15 a N_{-1}}{\sigma}\right)^{\frac{2}{3}}$, where $\omega_{\mathrm{rf}}=\left(\omega_{x} \omega_{\perp}^{2}\right)^{\frac{1}{3}}$ and $\sigma=\left(\hbar / M \omega_{\mathrm{rf}}\right)^{\frac{1}{2}}$ is the harmonic oscillator length. $E_{-1}=$ $\hbar \delta_{\mathrm{rf}}+\mu$ is the energy of the $m=-1$ state, $z_{\text {res }}=\frac{\hbar \delta_{\mathrm{rf}}+4 \mu / 7}{2 M g}$ is the resonance point where the output coupling takes place, $t_{\text {fall }}=\left[\frac{2}{g}\left(z-z_{\text {res }}\right)\right]^{\frac{1}{2}}$ is the falling time of the atoms from point $z_{\text {res }}$ and is constant for each particular atom laser, $l=\left(\frac{\hbar^{2}}{2 M^{2} g}\right)^{\frac{1}{3}}$ is the falling height such that $l \ll x_{0}, y_{0}, z_{0}$, the paramete $\xi_{\text {res }}=\frac{\left(z+z_{\text {res }}\right)}{l}$ provides a size scale of the trap, and $F\left(t, t_{\text {fall }}\right)$ describes the finite extent of the atom laser due to the finite coupling time. In the Thomas-Fermi (TF) approximation, the time-dependent ground state wave function $\phi_{-1}\left(x, y, z_{\text {res }}\right)$ is $[10,14]$ :

$$
\phi_{-1}\left(x, y, z_{\text {res }}\right)=\left(\frac{\mu}{U_{0}}\right)^{\frac{1}{2}}\left[1-\left(\frac{x}{x_{0}}\right)^{2}-\left(\frac{y}{y_{0}}\right)^{2}-\left(\frac{z}{z_{0}}\right)^{2}\right]^{\frac{1}{2}}
$$

where the BEC dimensions $x_{0}=\left(\frac{2 \mu}{M \omega_{x}^{2}}\right)^{\frac{1}{2}}$ and $y_{0}=z_{0}=$ $\left(\frac{2 \mu}{M \omega_{\perp}^{2}}\right)^{\frac{1}{2}}$. The time dependence of $\phi_{-1}\left(x, y, z_{\text {res }}\right)$ is contained in $\mu, x_{0}, y_{0}$, and $z_{0}$, which decrease with $N_{-1}(t)$, whereas the quantity $\left|\phi_{-1}\left(x, y, z_{\text {res }}\right)\right|^{2}$ corresponds to the trapped atomic population at the resonance point $z_{\text {res }}$. 


\section{THE CURRENT OF ATOMS WITH RF-FIELD SWITCHED ON}

As soon as the output coupling rf-field is switched on at $t=0$, the whole condensate undergoes an initial rapid oscillations damping through all the coupled states due to the escape of the untrapped atoms out of the trap under the influence of gravity within less than one Rabi cycle. At the same time, other atoms move to the resonance point replacing the leaving atoms, until a quasi-stationary state is reached. That is, the $m=-1$ condensate wave function decays slowly without oscillations while atoms coupled out of the condensate are expelled due to the mean-field potential and form a steady current. The kinetic energy term in equation (1) implies that a spatial transport of the atoms occurs, satisfying the conservation law:

$$
\frac{\partial\left|\psi_{0}(r, t)\right|^{2}}{\partial t}+\nabla \cdot J=0
$$

The current density is defined as:

$$
J=\frac{\hbar}{2 M i} \sum_{k} \sum_{k^{\prime}}\left(\psi_{0_{k}}^{*} \nabla \psi_{0_{k^{\prime}}}-\psi_{0_{k}} \nabla \psi_{0_{k^{\prime}}}^{*}\right)
$$

The state-vector for the beam of atoms propagating out of the trap can be written as:

$$
\psi_{0}(r, t)=\sum_{k} A_{k}(k) \exp [i(k \cdot r-\omega t)]
$$

We shall now calculate a general expression for the current density of the propagating beam of atoms. Considering rapid oscillations of the output coupling (i.e., neglecting the time dependence), the current density can be written using equation (10) as:

$$
J=\frac{\hbar}{2 M}\left\{2 \sum_{k} k\left|A_{k}\right|^{2}+\sum_{k^{\prime} \neq k} \sum_{k} k\left\{A_{k^{\prime}} A_{k}^{*} \exp \left[-i\left(k-k^{\prime}\right) \cdot r\right]+A_{k^{\prime}}^{*} A_{k} \exp \left[i\left(k-k^{\prime}\right) \cdot r\right]\right\}\right\}
$$

In the integral form, this equation reads as:

$$
J=\frac{\hbar}{2 M}\left\{2 \int_{k} k|A(k)|^{2} d k+\int_{k} \int_{k^{\prime} \neq k} k\left\{A\left(k^{\prime}\right) A^{*}(k) \exp \left[-i\left(k-k^{\prime}\right) \cdot r\right]+A^{*}\left(k^{\prime}\right) A(k) \exp \left[i\left(k-k^{\prime}\right) \cdot r\right]\right\} d k d k^{\prime}\right\}
$$

Taking:

$$
\tau=k-k^{\prime}
$$

then $d \tau=d k$ for a fixed $k^{\prime}$. Equation (13) describes a correlation between particles of momentum $k$ and $(k-\tau)$ for a given $k^{\prime}$. The beam of particles can be experimentally prepared in such a way that $A(k)$ is a real number. That is, for the initial condition $(t=0, r=0), A(k)$ has some definite values including zero. Accordingly, equation (12) becomes [8]:

$$
J=\frac{\hbar}{2 M}\left\{2 \int_{k} k|A(k)|^{2} d k+2 \int_{k^{\prime}} d k^{\prime} \int_{k} \operatorname{Re}\left[A\left(k^{\prime}-\tau\right) e^{-i\left(k^{\prime}-\tau\right) \cdot r}\right]\left[k A^{*}(k) e^{i k \cdot r}\right] d k\right\}
$$

which can be written as:

$$
J=\frac{\hbar}{2 M}\left\{2 \int_{k} k|A(k)|^{2} d k+2 \iint_{k} A_{k^{\prime}} A\left(k^{\prime}\right) A^{*}(k) k\left[\cos \left(k^{\prime}-k\right) \cdot r\right] d k d k^{\prime}\right\}
$$

This is the expression that we have been seeking for the current density of the propagating beam of atoms coupled out of

the condensate. 


\section{NUMERICAL MODEL FOR THE COHERENCE LENGTH OF ATOM LASER}

The second term in equation (15) describes a focusing phenomenon, both directional and longitudinal. For a pair of atoms travelling with a slight difference in $k$, the focusing along $z$ will correspond to those points where [8]:

$$
\left(k^{\prime}-k\right) \cdot z=2 n \pi, \quad n=1,2, \ldots
$$

The propagation vector $k$ is calculated using equation (6). With $\rho=|\psi|^{2}$, the particles velocity is:

$$
v=\frac{J}{\rho}=\frac{\hbar \sqrt{\xi_{\text {res }}}}{M l}=\frac{\hbar \sqrt{z+z_{\text {res }}}}{M l^{\frac{3}{2}}}
$$

Using De Broglie relation $p=\hbar k=m v$, the magnitude of $k$ will be:

$$
k=\frac{\sqrt{z+z_{\text {res }}}}{l^{\frac{3}{2}}}
$$

Using this expression of $k$ into equation (16) results in:

$$
z\left[\left(z+z_{\text {res }}\right)^{\frac{1}{2}}-\left(z^{\prime}+z_{\text {res }}\right)^{\frac{1}{2}}\right]=2 n \pi l^{\frac{3}{2}}
$$

Here $z-z^{\prime}$ represents the correlation length between two different points of the atom laser. Taking $z^{\prime}=z_{\text {res }}$, an expression for the coherence length of the atom laser, measured from the resonance point $z_{\text {res }}$, is found as follows [8]:

$$
z\left[\left(z+z_{\text {res }}\right)^{\frac{1}{2}}-\left(2 z_{\text {res }}\right)^{\frac{1}{2}}\right]=2 n \pi l^{\frac{3}{2}}
$$

Solving this equation numerically for $n=1$, we calculated the coherence lengths, $z$, of ${ }^{37} \mathrm{~K},{ }^{141} \mathrm{Ce}$, and ${ }^{249} \mathrm{Fm}$ atom lasers.

\section{RESULTS AND CONCLUSIONS}

We have developed a computer programme in FORTRAN to solve equation (20) numerically and calculate the coherence lengths, $z$, of ${ }^{37} \mathrm{~K},{ }^{141} \mathrm{Ce}$, and ${ }^{249} \mathrm{Fm}$ atom lasers. To verify our calculation method, we applied the programme first to three other atom lasers $\left({ }^{23} \mathrm{Na},{ }^{87} \mathrm{Rb}\right.$, and $\left.{ }^{7} \mathrm{Li}\right)$ of known coherence lengths in the literature $[8,9]$. This resulted in a very good agreement, encouraging us to calculate the coherence lengths of ${ }^{37} \mathrm{~K},{ }^{141} \mathrm{Ce}$, and ${ }^{249} \mathrm{Fm}$ atom lasers. Our results are listed in Table 1.

Table 1: Coherence lengths of some atom lasers obtained by this work and other researchers. The first three atoms were used to verify our calculation method.

\begin{tabular}{llll}
\hline & \multicolumn{3}{c}{ Coherence length $(\mu \mathrm{m})$} \\
\cline { 2 - 4 } & This work & Theoretical ${ }^{a}$ & Experimental $^{b}$ \\
\hline${ }^{23} \mathrm{Na}$ & 2.4814 & 2.4622 & 2.0 to 5.0 \\
${ }^{87} \mathrm{Rb}$ & 1.0246 & 1.0299 & \\
${ }^{7} \mathrm{Li}$ & 5.4803 & 5.4461 & \\
${ }^{37} \mathrm{~K}$ & 1.7722 & & \\
${ }^{141} \mathrm{Ce}$ & 0.7263 & & \\
${ }^{249} \mathrm{Fm}$ & 0.4968 & \\
${ }^{a}$ Ref. [8] \\
${ }^{b}$ Ref. [9]
\end{tabular}

We could not trace other values in the literature for these atom lasers in order to compare our results of the coherence lengths. Based on the validity of our calculation method and the excellent results that we have obtained for other atom lasers $\left({ }^{23} \mathrm{Na},{ }^{87} \mathrm{Rb}\right.$, and $\left.{ }^{7} \mathrm{Li}\right)$ of known coherence lengths, we conclude that our results for the coherence lengths of ${ }^{37} \mathrm{~K}$, ${ }^{141} \mathrm{Ce}$, and ${ }^{249} \mathrm{Fm}$ atom lasers are very reasonable.
[1] M. H. Anderson, J. R. Ensher, M. R. Matthews, and C. E. Wieman, E. A. Cornell, Science 269, 198 (1995).

[2] K. B. Davis, M.-O. Mewes, M. R. Andrews, N. J. van Druten, D. S. Durfee, D. M. Kurn, and W. Ketterle, Phys. Rev. Lett. 75, 3969 (1995).

[3] C. C. Bradley, C. A. Sackett, J. J. Tollett, and R. G. Hulet, Phys. Rev. Lett. 75, 1687 (1995).

[4] E. A. Cornell, C. E. Wieman, Rev. Mod. Phys. 74, 875 (2002)

[5] W. Ketterle, Rev. Mod. Phys. 74, 1131 (2002).

[6] M. R. Andrews, C. G. Townsend, H.-J. Miesner, D. S. Durfee, D. M. Kurn, and W. Ketterle, Science 275, 637 (1997).

[7] A. Rohrl, M. Naraschewski, A. Schenzle, and H. Wallis, Phys.Rev. Lett. 78, 4143 (1997).

[8] L. M. Castellanos, F. E. Lopez, quant-ph/0312168.

[9] M. Trippenbach, Y. B. Band, M. Edwards, M. Doery, P. S. Julienne, E. W. Hagley, L. Deng, M. Kozum, K. Helmerson, S. L.
Rolston, and W. D. Phillips, J. Phys. B: At. Mol. Opt. Phys. 33, 47 (2000).

[10] F. Gerbier, P. Bouyer, and A. Aspect, Phys. Rev. Lett. 86, 4729 (2001).

[11] M.-O. Mewes, M. R. Andrews, D. M. Kurn, D. S. Durfee, C. G. Townsend, and W. Ketterle, Phys. Rev. Lett. 78, 582 (1997).

[12] F. Dalfovo, S. Giorgini, L. Pitaevskii, and S. Stringari, Rev. Mod. Phys. 71, 463 (1999).

[13] H. Steck, M. Narachewski, and H. Wallis, Phys. Rev. Lett. 80, 1 (1998).

[14] R. J. Ballagh, K. Burnett, and T. F. Scott, Phys. Rev. Lett. 78 , 1607 (1997).

[15] R. Ballagh, C. M. Savage, Mod. Phys. Lett. B 14 Suppl. Issue 1 153 (2000). 